\title{
Modelación de riesgo operacional causado por factores demográficos ${ }^{*}$
}

\author{
Diego Fernando Manotas* \\ Inés María Ulloa ${ }^{* * *}$ \\ Jorge Mario Uribe $e^{* * * *}$
}

Recibido: 12/12/2014 - Aceptado: 09/12/2015

DOl: 10.22395/rium.v15n29a7

\begin{abstract}
Resumen
En el presente artículo de investigación, se propone una metodología para medir el riesgo financiero en empresas no financieras expuestas ante variables como tasas de mortalidad o tasas de morbilidad. La metodología desarrollada incorpora elementos de la literatura actuarial, la economía financiera y la teoría de cópulas. La metodología se centra en la medición del riesgo subyacente a factores demográficos y permite minimizar los requerimientos de información para su estimación. Finalmente, la metodología se ilustra con su aplicación a la medición de riesgo financiero de una empresa de auxilios funerarios.
\end{abstract}

Palabras clave: riesgo financiero, factores demográficos, valor en riesgo, valor en riesgo condicional.

* El artículo se deriva del proyecto de investigación "Estimación de reserva técnica por riesgo de negocio", financiado por la Cooperativa Servivir en Colombia y ejecutado entre los meses de junio a diciembre de 2014 .

** Profesor titular Escuela de Ingeniería Industrial, Universidad del Valle. Ph. D. Ingeniería - Universidad del Valle, M. Sc. Gestión Financiera, Universidad de Chile. Ingeniero Industrial, Universidad del Valle. diego. manotas@correounivalle.edu.co. Ciudad Universitaria, Calle 13 N.1100-00 Cali, Tel. 5723212167 Ext. 104 Fax 57-2-3398462

*** Profesora asociada, Departamento de Economía, Universidad del Valle. Diplom Volkswirtin, Universität Mannheim. Economista, Universidad del Valle. inesmaria.ulloa@correounivalle.edu.co. Ciudad Universitaria, Calle 13 N. ${ }^{\circ}$ 100-00 Cali, +57 23212322

**** Profesor asociado Departamento de Economía, Universidad del Valle. Master of Research, European University Institute. Magíster en Economía, Universidad de los Andes. Economista, Universidad del Valle. jorge.uribe@ correounivalle.edu.co. Ciudad Universitaria, Calle 13 N. ${ }^{\circ}$ 100-00 Cali, +57 23212322 


\title{
Modeling operational risk caused by demographic factors
}

\begin{abstract}
In this research paper, we propose a methodology to measure the financial risk in non-financial companies exposed to variables such as mortality and morbidity rates. The developed methodology includes elements from actuarial literature, financial economics and copulation theory. The methodology focuses on the measurement of the underlying risk to demographic factors and allows to simplify the information needed for its calculation. Finally, the methodology is validated by applying the financial risk measurement on a funeral insurance company.
\end{abstract}

Key words:financial risk, demographic factors, value at risk, value at conditional risk. 


\section{INTRODUCCIÓN}

La medición del riesgo operacional es una de las áreas críticas de los procesos de gestión de riesgo financiero. El riesgo operacional obedece a factores cuya cuantificación se hace muy compleja debido a la carencia de información de soporte. Desde hace algunos años, se ha desarrollado un conjunto significativo de instrumentos técnicos, útiles para medir distintos tipos de riesgo. Este avance se observa en mayor medida en el área financiera, en la cual diferentes técnicas estadísticas y econométricas han sido adoptadas para contribuir con los procesos de medición, y con la toma de decisiones óptimas bajo incertidumbre, por parte de las empresas [1]. De esta forma, es posible encontrar técnicas econométricas útiles para medir diferentes tipos de riesgo financiero como mercado, liquidez, crédito, operacional o, incluso, el riesgo sistémico, que engloba aspectos de todos los anteriores [2].

Este documento plantea un avance en esta dirección, ya que estudia las fuentes de riesgo relevantes para empresas vinculadas en su operación con variables de tipo demográfico.

La estrategia se concentra: en la identificación de los factores de riesgo pertinentes para el negocio; la modelación estadística adecuada del riesgo marginal implícito en cada uno de estos factores; la modelación completa de las relaciones de codependencia que pueden existir entre ellos y, finalmente, el mapeo desde el dominio de los factores de riesgo hacia los indicadores financieros de las empresas.

La metodología se ilustra mediante su aplicación a la medición del riesgo de mortalidad, al que se encuentra expuesta una empresa de seguros funerarios, cuyos egresos futuros de operación están determinados por la materialización de eventos fúnebres dentro de la población afiliada. Se tiene información sobre esa población discriminada en rangos de edad y género, y se requiere analizar el comportamiento de la reserva técnica patrimonial asociada con la operación de la empresa. La propuesta metodológica involucra el uso de distintos modelos propios de la ciencia actuarial, tales como el modelo demográfico de Lee - Carter [3], así como de otros pertenecientes al ámbito de la econometría financiera, como el valor en riesgo (VaR), el valor en riesgo condicional (CoVaR) y la estimación de relaciones de dependencia mediante cópulas bivariadas.

En relación con la modelación de riesgos demográficos, la literatura es extensa. Incluso dentro del campo específico del pronóstico de las tasas de mortalidad existen abundantes vertientes. Aplicaciones del modelo de Lee-Carter en casos latinoamericanos se pueden encontrar por ejemplo en México [4] y en Chile [5]. 


\section{METODOLOGÍA}

La metodología propuesta consta de 4 pasos: identificación de factores de riesgo, modelación de los comportamientos univariados, estimación de las relaciones de dependencia y mapeo desde los factores de riesgo a los indicadores financieros de la empresa.

\subsection{Identificación de los factores de riesgo}

En el caso ilustrativo, considerado en el presente artículo, se tiene que los principales egresos futuros de la empresa dependen del número de fallecimientos registrados en la población afiliada. Entre mayor sea el número de fallecimientos, mayor será el desembolso en términos de auxilios funerarios. Por lo tanto, no existen variables de control propiamente dichas. Por una parte, se tiene que la pérdida esperada depende de las tasas de mortalidad específicas, por rangos de edad y sexo, de los grupos de personas afiliados a la entidad. Por otro lado, se sabe que tal pérdida depende de la población afiliada al fondo, discriminada en las mismas categorías de las tasas de mortalidad. Para facilitar el cálculo, esta población se supone constante a lo largo del año. De esta forma, la pérdida esperada está dada por

$$
P E=\sum_{i=1}^{N} T m_{i} P_{i}
$$

En donde, la pérdida esperada, $P E$, depende linealmente de las tasas de mortalidad específicas, $T m_{i}$, en cada grupo etario por sexo. Depende también de la población afiliada, discriminada una vez más por rango de edad y sexo, $P_{i}$. En total se identifican 34 factores de riesgo: tasas de mortalidad para hombres y mujeres en grupos de edades: menores a 1 año, entre 1-4 años,..., 70-74 años y de 75 años en adelante.

\subsection{Modelación de los comportamientos univariados}

Cada uno de los 34 factores de riesgo debe ser pronosticado, para poder así estimar su participación dentro de la pérdida esperada de la compañía. Para tal fin, se exploran, en este paso, dos metodologías alternativas: una de ellas el modelo de Lee \& Carter [3], y otra los modelos de series de tiempo univariados tradicionales, ARIMA (autorregresivos integrados de medias móviles).

El modelo de Lee-Carter es un modelo estadístico ampliamente utilizado en la literatura sobre demografía y actuaría, para la estimación y pronóstico de tasas específicas de mortalidad y tablas de vida [7-10]. Se ha convertido en una herramienta indipensable dentro de la formulación de políticas públicas relacionadas con la expectativa de vida de una población específica y el pronóstico de sus tasas de mortalidad. 
Formalmente, sea $\log m_{x t}$ el logaritmo natural de la tasa de mortalidad específica del grupo $x$ en el período $t$. Se tiene que el modelo puede ser estructurado en términos de $a \mathrm{y} \mathrm{b}$ en la dimensión etaria, y un vector $k$ a lo largo de la dimensión temporal, de forma tal que:

$$
\log m_{x t}=a_{x}+b_{x} k_{t}+\varepsilon_{x t}
$$

Adicionalmente deben cumplirse las restricciones de que $\sum_{x} b_{x}=1$ y $\sum_{t} k_{t}=0$ de manera que las $a_{x}$ son promedios temporales simples de los logaritmos de las tasas de mortalidad específicas, y los parámetros del sistema están plenamente identificados.

La estrategia de estimación consiste en utilizar una descomposición de valor singular (SVD), puesto que el método de mínimos cuadrados ordinarios no puede ser aplicado directamente en la ecuación (2), ya que el índice $k_{t}$ debe ser también estimado en el proceso. De esta forma, $k_{t}$ es un índice del nivel de mortalidad en el tiempo, mientras que $a_{x}$ indica la forma general de esta mortalidad a lo largo del espectro de los distintos rangos de edad. $b_{x}$, por su parte, mide la sensibilidad de la tasa de mortalidad específica ante cambios en $k_{t}$ (ya que $d \log \left(m_{x t}\right) / d t=b_{x} d k / d t$ ).

La popularidad del modelo con respecto a modelos tradicionales de series de tiempo ARIMA se debe a la inclusión del término $k_{t}$ que es común a todas las tasas. Este solo varía en el tiempo y no tranversalmente. De esta forma, se reduce la cantidad de parámetros a ser estimados, a la vez que impone un comportamiento acompasado entre las tasas, impididiendo pronósticos en intervalos largos de tiempo dispersos.

Dado que en el ejercicio presente no es de interés la construcción de tablas de vida a lo largo de dominios prolongados de tiempo, es posible utilizar modelos ARIMA tradicionales para el pronóstico de las tasas de mortalidad individuales, a manera de comparación, para dar robustez a los hallazgos.

En este caso se aplicó un modelo ARIMA a los logaritmos de la serie de tiempo de cada una de las tasas específicas de mortalidad de forma tal que

$$
\log m_{x t}=\beta_{x}+\sum_{i=1}^{p} \rho_{i} \log m_{x t-i}+\sum_{j=1}^{q} \theta_{j} \varepsilon_{x t-i}+\varepsilon_{x t}
$$

Se utilizan logaritmos para garantizar la no-negatividad del pronóstico. La ecuación (3) se estima para cada grupo poblacional. $\beta_{x}$ el intercepto de cada regresión. Por su parte, $\rho_{i}$ y son los coeficientes autorregresivos y de medias móviles del sistema, respectivamente. Los números $i$ y $j$ son determinados a través de comparación de modelos, proceso en el cual se selecciona el modelo con un menor criterio de información 
bayesiano (BIC). $\varepsilon_{x t}$ es un término de perturbación ruido blanco. Cuando se detecta la presencia de raíces unitarias se procede a la diferenciación de la variable, para lograr la estacionariedad.

\subsection{Modelación de las relaciones de dependencia}

Hasta aquí solo se han considerado los comportamientos univariados de los factores de riesgo. Si bien el método de Lee-Carter impone una dependencia lineal en la parte de baja frecuencia de los espectros de las series, aún en este caso las relaciones de dependencia no lineales se dejan por fuera del análisis, y también así las que se presentan en el resto del espectro. Estas relaciones son de gran importancia en el análisis, puesto que un incremento abrupto y conjunto en las tasas de mortalidad podría configurar un escenario de operación de gran estrés financiero, y podría llevar a la quiebra.

Una forma particularmente conveniente y completa de modelar las relaciones de dependencia entre las tasas de mortalidad en este tipo de problemas la constituye la estimación de tales relaciones mediante cópulas. Formalmente, una cópula es una función de distribución multivariada tal que

$$
\mathrm{C}:[0,1]^{\mathrm{N}} \rightarrow[0,1]
$$

Donde $\mathrm{N}$ son el número de factores de riesgo descritos antes y $C$ denota la cópula.

En términos generales el teorema de Sklar (1959) plantea que si $F$ es una función de distribución multivariada con marginales $F_{l}, \ldots, F_{N}$ (no necesariamente continua), entonces, existe una cópula $C:[0,1]^{n} \rightarrow[0,1]$ tal que, para todo $\mathrm{z}_{1}, \ldots, \mathrm{z}_{\mathrm{N}}$ en $\mathrm{R}=[-\infty, \infty]$ :

$$
\mathrm{F}\left(\mathrm{z}_{1}, \ldots, \mathrm{z}_{\mathrm{N}}\right)=\mathrm{C}\left(\mathrm{F}_{1}\left(\mathrm{z}_{1}\right), \ldots, \mathrm{F}_{\mathrm{N}}\left(\mathrm{z}_{\mathrm{N}}\right)\right)
$$

Si las marginales son continuas, $C$ es única.

Cabe anotar que la cópula no se encuentra definida sobre las tasas de mortalidad directamente, ni sobre las series filtradas a través del modelo ARIMA o el Modelo de Lee-Carter. Primero es necesario construir una pseudo-muestra a saber:

$$
F_{i}\left(z_{i}\right)=u_{i} \quad \mathrm{i}=1, . ., \mathrm{N}
$$

Donde $z_{i}$ son los residuales de la regresión en las ecuaciones 2 o 3. Para construir $\mu$, que es el vector con componentes del tipo $\mu_{i}$, en este estudio se hace uso de las distribuciones empíricas acumuladas de probabilidad, asociadas con cada factor de 
riesgo. Una consideración adicional que se debe tener en cuenta es que al trabajar con muchos factores de riesgo la estimación de una cópula multivariada no es recomendable; de hecho, construir modelos de este tipo es un problema de reconocida dificultad [13]. Una alternativa de amplio uso en la literatura reciente es el uso de pair-copulas (una estructura jerárquica de cópulas bivariadas).

Siguiendo este esquema como en [13], se muestra que al mismo tiempo que se mantiene la lógica de elaborar relaciones complejas a través de bloques individuales simples, se minimiza la cantidad de supuestos de independencia condicional, que muchas veces deben ser impuestos, al utilizar otros caminos en el proceso de estimación.

Formalmente, la función de densidad, asociada con la función de distribución acumulada en (5), puede ser factorizada así:

$$
f\left(\mathrm{z}_{1}, \ldots, \mathrm{z}_{\mathrm{N}}\right)=f_{n}\left(z_{N}\right) \cdot f\left(\mathrm{z}_{\mathrm{N}-1} \mid z_{N}\right) \cdot f\left(\mathrm{z}_{\mathrm{N}-2} \mid z_{N-1}, z_{N}\right) \cdots f\left(z_{1} \mid z_{2}, \ldots, z_{N}\right)
$$

A su vez, cada término en (7) puede ser descompuesto en una cópula bivariada, multiplicada por una densidad marginal condicional. Usando la fórmula general se tiene que:

$$
f(z \mid \boldsymbol{v})=c_{z v_{j}} \mid \boldsymbol{v}_{-j}\left\{F\left(z \mid \boldsymbol{v}_{-j}\right), F\left(v_{j} \mid \boldsymbol{v}_{-j}\right)\right\} \cdot f\left(z \mid \boldsymbol{v}_{-j}\right)
$$

para un vector $\mathrm{N}$-dimensional $v$. Aquí $\mathrm{v}_{j}$ es un componente escogido arbitrariamente de $v . v_{-j}$ denota el vector- $v$, excluyendo ese componente. Por su parte, $c_{z v_{j}}$ es una cópula bivariada (cópula-par) entre $z$ y $v_{\mathrm{j}}$. En conclusión, tal y como se señala en [13], bajo condiciones de regularidad apropiadas, una densidad multivariada puede ser expresada como el producto entre cópulas-par, actuando sobre diferentes distribuciones de probabilidad condicionales. También es claro que la construcción es, por naturaleza, iterativa, y que, dada una factorización específica, existen muchas re-parametrizaciones con las cuales se puede lograr este objetivo.

Estas diferentes construcciones de cópulas-par disponibles pueden ser descritas a partir de [14] en un modelo gráfico denominado vine regular. La clase de vine regular es amplia. En este estudio se sigue una de las posibilidades de mayor uso, conocida como D-vine [15]. Cada modelo señala una forma específica de descomponer la densidad.

La figura 1 describe una descomposición D-vine de cinco dimensiones. Consta de cuatro árboles $T_{j}, j=1, \ldots, 4$. El árbol $T_{j}$ tiene $6-j$ nodos y $5-j$ ejes. Cada eje corresponde a una densidad de cópula-par, y el rótulo del eje corresponde al subíndice de la densidad bivariada, e.g. el eje 25|34 corresponde a la cópula $c_{25 \mid 34}\left({ }^{\circ}\right)$. 


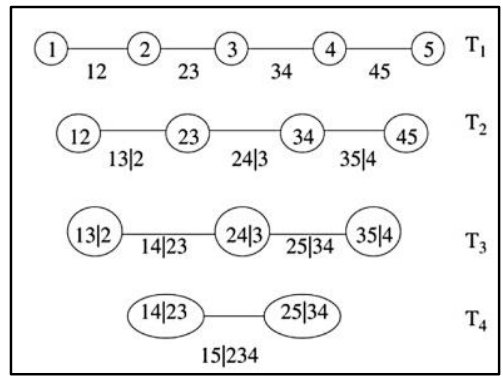

Figura 1. D-vine con 5 variables, 4 árboles y 10 ejes Fuente: Aas et al. (2009), p. 184

En [14] se proporciona la densidad de una distribución N-dimensional en términos de un vine-regular. De esta forma la densidad de $f\left(z_{1}, \ldots, z_{N}\right)$ puede ser escrita como:

$$
\begin{aligned}
& \prod_{k=1}^{N} f\left(z_{k}\right) \prod_{j=1}^{N-1} \prod_{i=1}^{N-j} c_{i, i+j \mid i+1, \cdots, i+j-1} \\
& \left\{F\left(z_{i} \mid z_{i+1}, \ldots, z_{i+j-1}\right), F\left(z_{i+j} \mid z_{i+1}, \ldots, z_{i+j-1}\right)\right\}
\end{aligned}
$$

Donde los índices $j$ identifican los árboles, mientras que los índices $i$ denotan los ejes en cada árbol. En los D-vine, ningún nodo en ningún árbol conecta con más de dos ejes. En [13] se proveen los pasos necesarios para realizar simulaciones usando las cópulas estimadas en el constructo D-vine. Este método es necesario para predecir el número de fallecimientos que se puede presentar en escenarios de estrés en la operación de la empresa.

\subsection{Mapeo desde factores de riesgo hacia indicadores financieros}

Una vez modelados los factores de riesgo, es posible obtener por simulación diferentes escenarios de operación de la empresa en términos del número de eventos que se pueden registrar en el siguiente año de operación. En particular se utilizan, además de la pérdida esperada, dos indicadores de amplio uso en la ingeniería financiera, como lo son el valor en riesgo (VaR) y el valor en riesgo condicional (CoVaR) $[2,6,16]$.

\subsubsection{Resumen del algoritmo}

i) Se simulan $M$ escenarios de operación de la empresa (1.000 por ejemplo), en términos de las tasas de mortalidad que pudieren observarse.

ii) Se toma el escenario medio (que corresponde al pronóstico de la pérdida, pérdida esperada),

iii) Se calcula un escenario extremo, al $(1-\alpha) \%$ de confianza $\left(\operatorname{VaR}_{1-\alpha}\right)$ y 
iv) Se construye un escenario de pérdida sistémica, asociado con el promedio de todos los escenarios por encima del $\left(V_{a} R_{1-\alpha}\right)$, denominado $\mathrm{CoVaR}_{1-\alpha}$.

v) En cada caso se multiplican las tasas correspondientes por la población afiliada por sexo y rango de edad, de la empresa, la cual se supone fija durante el año de operación.

$$
\begin{gathered}
P E=\sum_{i=1}^{N} T M_{i} \cdot P_{i} \\
\operatorname{VaR}_{1-\alpha}=\sum_{i=1}^{N} T M_{i, 1-\alpha} \cdot P_{i} \\
\operatorname{CoVaR}_{1-\alpha}=\int_{1-\alpha}^{1} \sum_{i=1}^{N} T M_{i, 1-\alpha} \cdot P_{i} d m / \alpha
\end{gathered}
$$

Donde $P_{i}$ es la población perteneciente a cada rango de edad y sexo. $T M_{i}$ es la tasa específica de mortalidad por rango de edad y sexo.

\section{ANÁLISIS DE RESULTADOS}

Para estimar la dinámica de las tasas de mortalidad es necesaria una serie de tiempo de cada tasa con una longitud considerable. Las tasas de mortalidad observadas en la población afiliada de las empresas son generalmente muy pocas, por lo que es mejor recurrir a las tasas de mortalidad de la zona geográfica de operación de la empresa, en este caso, la ciudad de Santiago de Cali. En esta ciudad se desarrolla la gran mayoría de las operaciones de la empresa y en donde se emplaza más del $80 \%$ de su población de clientes.

\subsection{Datos}

Los datos de las tasas de mortalidad por géneros y según los rangos de edad especificados se obtuvieron directamente de la Secretaría de Salud Municipal de Cali, con una frecuencia anual desde 1985 hasta 2012.

La empresa, por su parte, proporcionó información sobre la población afiliada, según género, fecha de nacimiento y defunción. Con esta información fue posible caracterizar la población, tarea para la cual se hizo uso de 74.644 registros. La figura 2 describe en términos generales a la población afiliada, y la figura 3, las defunciones registradas al año 2013 dentro de la población afiliada de la empresa en el mismo año. 


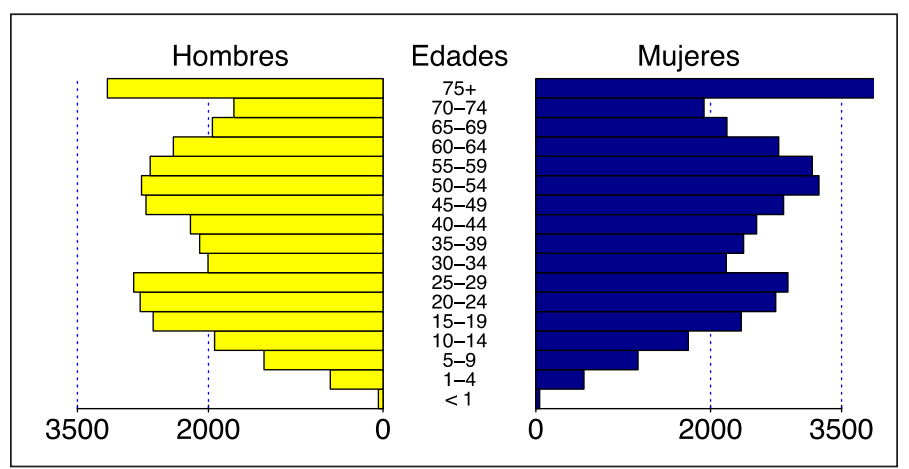

Figura 2. Población afiliada por rango de edad y sexo. Año 2014 Fuente: elaboración propia, con base en información suministrada por la empresa

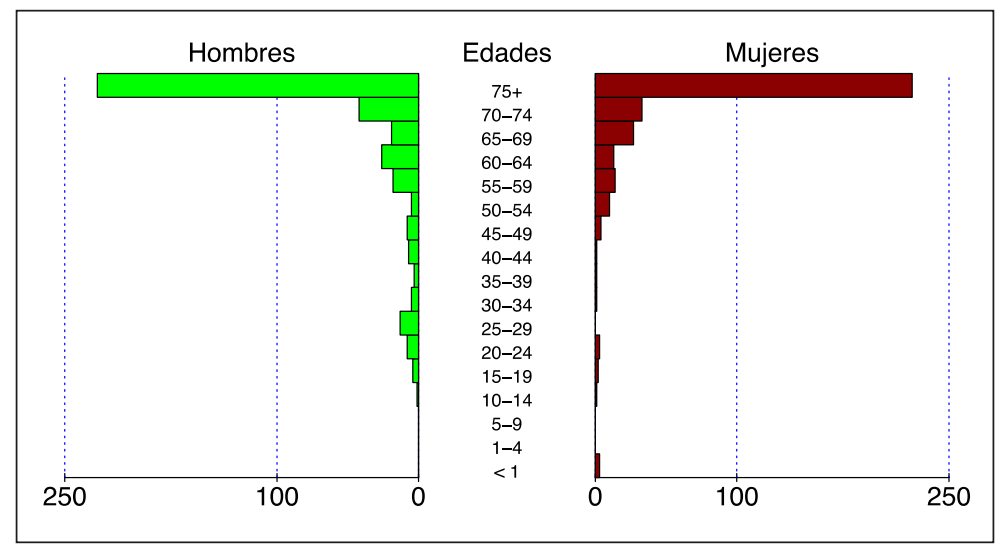

Figura 3. Defunciones registradas en el año 2013

Fuente: elaboración propia, con base en información suministrada por la empresa

Como se puede observar, debido al tipo de negocio de la empresa, tanto la población general afiliada, como el número de defunciones registrado se encuentran concentrados en los rangos de edad más altos (entre 70 y 74 años, y más de 75 años). El número de hombres y mujeres es, por su parte, equiparable. Esta mayor concentración se tiene en cuenta en el cálculo de la pérdida esperada, el $\operatorname{VaR}$ y el $\mathrm{CoVaR}$, en las fórmulas (10) a (12), ya que, a pesar de que la tasa de mortalidad pronosticada debe hacer uso de la información para la ciudad, el cálculo de los indicadores se realiza multiplicando por las poblaciones específicas de la empresa.

Las tasas de mortalidad por sexo y rango de edad para Cali, desde 1985 a 2012, que constituyen la muestra de estudio, se presentan en la figura 4. 


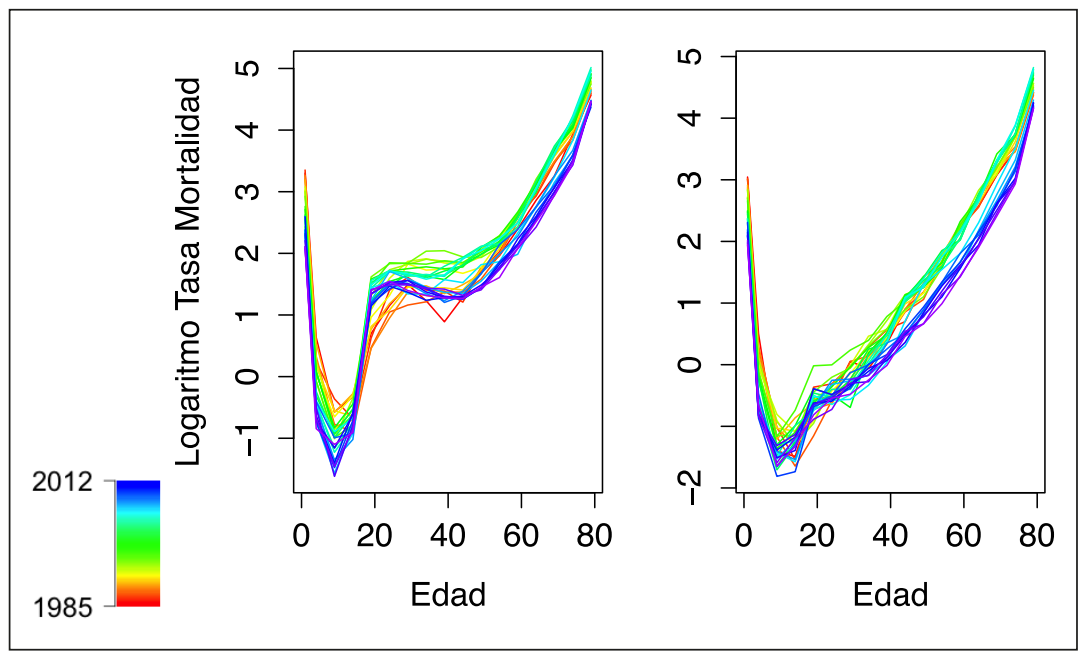

Figura 4. Logaritmo de las tasas de mortalidad de hombres (izq) mujeres (der), por rango de edad: 1985-2012

Fuente: elaboración propia

Como se puede observar, tanto para hombres como para mujeres, las tasas de mortalidad han presentado importantes reducciones durante el período de estudio, lo cual está relacionado con el proceso de desarrollo económico y las consecuentes mejoras en términos de morbilidad y mortalidad que este proceso conlleva.

\subsection{Resultados Modelo ARIMA}

Los modelos ARIMA univariados, ajustados sobre el logaritmo de las tasas de mortalidad, se hicieron con el paquete de R, auto.arima. Se escogió el mejor modelo siguiendo el criterio bayesiano (BIC), es decir, se escogió el modelo que minimizara tal estadístico en cada caso. Este criterio permite tener en cuenta, no solo el ajuste del modelo dentro de la muestra, sino que incorpora cierta penalización por el número de grados de libertad consumidos en la estimación (para minimizar la probabilidad de sobreajuste en muestra).

Los modelos escogidos de esta forma tienden a ser más parsimoniosos que los especificados siguiendo criterios alternativos.

Los estadísticos asociados con estas estimaciones no se reportan en este documento, pero están disponibles a petición del interesado. En la tabla 1 se presentan las tasas de mortalidad pronosticadas a un año por grupo de edad. 
Diego Fernando Manotas - Inés María Ulloa - Jorge Mario Uribe

Tabla 1. Pronósticos por rango de edad y sexo ARIMA. Tasas de mortalidad 0/00

\begin{tabular}{|c|c|c|c|c|c|c|c|c|c|}
\hline \multirow{4}{*}{ Hombres } & $<1$ & $1-4$ & $5-9$ & $9-14$ & $15-19$ & $20-24$ & $25-29$ & $30-34$ & $35-39$ \\
\cline { 2 - 10 } & 7,69 & 0,45 & 0,27 & 0,45 & 4,03 & 4,7 & 4,36 & 3,83 & 3,63 \\
\cline { 2 - 11 } & & $40-44$ & $45-49$ & $50-54$ & $55-59$ & $60-64$ & $65-69$ & $70-74$ & $>75$ \\
\cline { 2 - 10 } & & 3,61 & 4,27 & 5 & 8,12 & 12,37 & 18,15 & 31,49 & 87,01 \\
\hline \multirow{4}{*}{ Mujeres } & $<1$ & $1-4$ & $5-9$ & $9-14$ & $15-19$ & $20-24$ & $25-29$ & $30-34$ & $35-39$ \\
\cline { 2 - 11 } & 6,71 & 0,44 & 0,2 & 0,28 & 0,53 & 0,65 & 0,7 & 0,8 & 1,06 \\
\cline { 2 - 11 } & & $40-44$ & $45-49$ & $50-54$ & $55-59$ & $60-64$ & $65-69$ & $70-74$ & $>75$ \\
\cline { 2 - 10 } & & 1,58 & 1,98 & 2,69 & 4,55 & 6,7 & 11,16 & 18,43 & 64,01 \\
\hline
\end{tabular}

Fuente: elaboración propia

Con los residuales de los modelos ARIMA se construyó la pseudo-muestra. Se contrastó la hipótesis de que tal pseudo-muestra descrita en la ecuación (6) estaba bien construida, mediante un estadístico de Kolmogorov-Smirnov. En ningún caso se rechaza la hipótesis nula de que la pseudo-muestra se distribuye uniforme entre 0 y 1 .

El procedimiento de ajuste de la cópula en la pseudo-muestra implica la elección de la estructura que más se acople a los datos en cada paso de la iteración. Esto se hizo, una vez más, automáticamente, con la ayuda del paquete de R, CD Vine. Se consideraron 40 categorías disponibles.

Finalmente, se simularon 1000 escenarios, de los cuales se extrajeron el VaR y el CoVaR al $90 \%$ de confianza, construidos después de multiplicar cada uno de los escenarios simulados por la población expuesta. La síntesis de los escenarios se presenta en la figura 5.

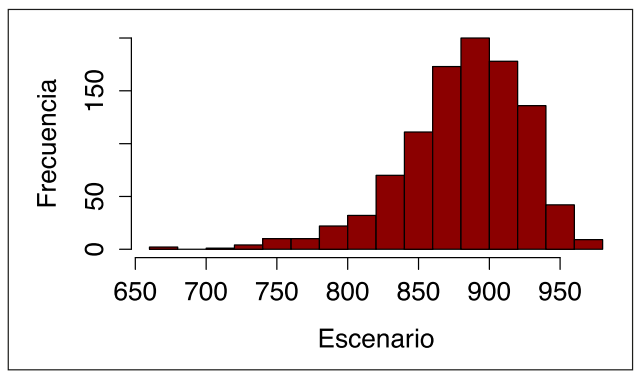

Figura 5. Histograma de los escenarios proyectados del modelo ARIMA Fuente: elaboración propia 
Para el caso del modelo ARIMA la pérdida esperada, en términos de número de fallecimientos, a un año, ascendió a 882. El VaR, con un $90 \%$ de confianza, se ubicó en 930, y el CoVaR, en 944.

\subsection{Resultados modelo Lee- Carter}

El modelo de Lee-Carter se ajustó con ayuda del paquete en R Demography. En la tabla 2 se presentan las tasas de mortalidad pronosticadas a un año por grupo de edad.

Tabla 2. Pronósticos modelo de Lee-Carter. Tasas de mortalidad 0/00

\begin{tabular}{|c|c|c|c|c|c|c|c|c|c|}
\hline \multirow{4}{*}{ Hombres } & $<1$ & $1-4$ & $5-9$ & $9-14$ & $15-19$ & $20-24$ & $25-29$ & $30-34$ & $35-39$ \\
\cline { 2 - 10 } & 7,81 & 0,42 & 0,22 & 0,41 & 3,92 & 4,69 & 4,19 & 3,68 & 3,46 \\
\cline { 2 - 10 } & & $40-44$ & $45-49$ & $50-54$ & $55-59$ & $60-64$ & $65-69$ & $70-74$ & $>75$ \\
\cline { 2 - 10 } & & 3,47 & 4,20 & 5,69 & 7,76 & 11,91 & 18,74 & 31,20 & 81,06 \\
\hline \multirow{4}{*}{ Mujeres } & $<1$ & $1-4$ & $5-9$ & $9-14$ & $15-19$ & $20-24$ & $25-29$ & $30-34$ & $35-39$ \\
\cline { 2 - 10 } & 6,68 & 0,38 & 0,19 & 0,25 & 0,49 & 0,57 & 0,67 & 0,77 & 1,01 \\
\cline { 2 - 10 } & & $40-44$ & $45-49$ & $50-54$ & $55-59$ & $60-64$ & $65-69$ & $70-74$ & $>75$ \\
\cline { 2 - 10 } & & 1,44 & 2,08 & 3,05 & 4,20 & 6,66 & 11,59 & 19,54 & 63,98 \\
\hline
\end{tabular}

Fuente: elaboración propia

La síntesis de los escenarios simulados con este modelo se presenta en la figura 6.

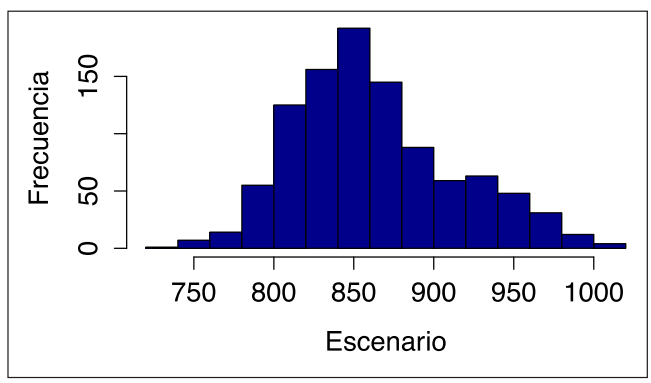

Figura 6. Histograma de los escenarios proyectados del modelo ARIMA Fuente: elaboración propia

Para el caso del modelo de Lee-Carter la pérdida esperada, en términos de número de fallecimientos, a un año, ascendió a 863. El VaR con un $90 \%$ de confianza se ubicó en 938, y el CoVaR, en 962. 


\subsection{Análisis de la reserva técnica de operación}

Para la valoración de la reserva técnica de operación, que se desprende de la anterior metodología, se procedió al análisis de los estados financieros de la entidad a diciembre 31 de 2013. Con esta información se estimó el indicador de utilidad para cobertura de servicio (UCS). Este se obtiene como la diferencia entre los ingresos operacionales y los gastos de funcionamiento de la entidad: $\mathrm{UCS}=$ Ingresos operacionales - Gastos de personal - Gastos Generales. El indicador de utilidad para cobertura de servicio permite estimar la capacidad de atender los auxilios y servicios funerarios comprometidos a partir de su ejercicio anual. El resultado deseable en términos de este indicador es que la utilidad para cobertura de servicio de un determinado período haya sido suficiente para cubrir los costos y auxilios funerarios del mismo período, sin necesidad de recurrir a reserva alguna.

Al observar el período 2008-2013, (figura 7) se puede observar que la cobertura de los servicios y auxilios funerarios se ha realizado básicamente con los recursos provistos por el propio negocio después de descontar los gastos de funcionamiento del mismo. Esta situación ha permitido que la entidad pueda fortalecer su reserva técnica a lo largo de los años.

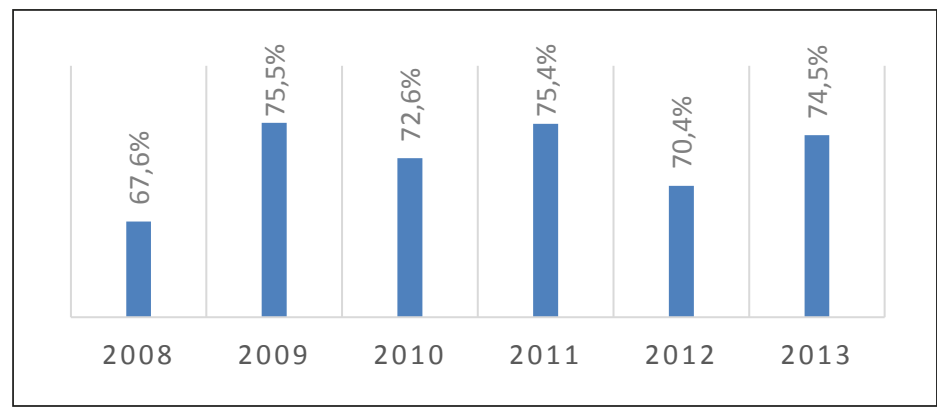

Figura 7. Indicador Costo de servicios funerarios / UCS Fuente: elaboración propia

Posteriormente se simularon escenarios de operación de la empresa, mediante los pronósticos del modelo de Lee-Carter (CoVaR), y se evaluaron costos promedio de eventos entre $\$ 1400000$ y $\$ 2000000$ de pesos. Para los escenarios de riesgo extremo propuestos, el costo del servicio funerario podría oscilar entre \$1.3 millones y \$1.9 millones de pesos, lo cual implicaría que aún, en escenarios extremos, se puede cubrir el servicio funerario con la utilidad para cobertura de servicio.

Un análisis aún más extremo se puede llevar a cabo utilizando el CoVaR estimado, en varios años de forma consecutiva. Para este escenario se considera un costo medio de servicio de $\$ 1.810 .750$. Con este valor se estima el costo total anual de auxilio 
funerario, el cual se compara contra la utilidad de cobertura de servicio del mismo año. Los desfases son cubiertos por la reserva de la entidad que a diciembre de 2013 ascendía a \$2.307 millones de pesos. Con estas situaciones extremas recurrentes, el fondo de imprevistos de la empresa se estaría agotando entre el año 2018 y el 2019, sin considerar ningún tipo de realimentación financiera.

\section{CONCLUSIONES}

Se propone una metodología para estimar el riesgo financiero de una empresa expuesta ante factores de tipo demográfico. Se identifica en las tasas de mortalidad por sexo y rangos de edad como el principal factor de riesgo del negocio y para su modelación se recurre a modelos por factores como el de Lee-Carter y a cópulas bivariadas. Se simulan varios escenarios de operación y financieros de una empresa a manera de ejemplo, con datos reales y, de esta forma, se hace posible estimar la reserva técnica óptima de operación de la entidad y el tiempo en que se agotaría tal reserva.

Esta estrategia de modelación tiene en cuenta diferentes hechos estilizados de las tasas de mortalidad, como el hecho de que no son estacionarias en media, y los patrones de dependencia temporal y transversal en el conjunto de tasas de mortalidad. Los patrones de dependencia transversal son capturados mediante cópulas bivariadas, mientras que los de dependencia temporal, mediante modelos tradicionales de series de tiempo univariadas. Estos últimos son aplicados sobre las diferencias logarítmicas de las tasas de mortalidad. La presente metodología tiene aplicaciones tanto para el pronóstico directo de diferentes escenarios de operación de empresas expuestas a riesgos de mortalidad y longevidad, como a la construcción de intervalos de confianza para el pronóstico de tasas de mortalidad en demografía y actuaría.

Los resultados señalan que las estrategias adoptadas históricamente por la empresa para la constitución de la reserva técnica han sido efectivas en la medida en que se ha logrado contar con un mecanismo de cobertura eficiente ante los siniestros normales que se han presentado.

\section{REFERENCIAS}

[1] C. W. J. Smith, "Managing Corporate Risk", in Handbook of Corporate Finance: Empirical Corporate Finance, Vol. 2., North-Holland, Elsevier, 2009, pp. 539-556.

[2] A. J. McNeil, R. Frey, and P. Embrechts, Quantitative Risk Management: concepts, techniques and tools. Princeton University Press, 2005.

[3] R. D. Lee and L. R. Carter, "Modeling and Forecasting U. S . Mortality", J. Am. Stat. Assoc., vol. 87, N. ${ }^{\circ} 419$, pp. 659-671, 1992. 
[4] J. Aburto, "Estimación de la mortalidad mexicana por el método de Lee-Carter y por el modelo aditivo doble multiplicativo propuesto por wilmoth", Dirección de Análisis Demogr. XI Reunión Nacional de Investigación Demográfica en México, México, 2012.

[5] J. Alonso, D. Tuesta, D. Torres, and B. Villamide, "Proyecciones de tablas generacionales dinámicas y riesgo de longevidad en Chile”, Madrid, 13/12, 2013.

[6] P. F. Christoffersen, Elements of financial risk management. Elservier- Academic Press, 2012.

[7] A. Deaton and C. Paxson, "Mortality,income and income inequality over time in Britain and the United States", in Perspectives on the Economics of Aging, Chicago: University of Chicago Press, 2004.

[8] F. W. Hollmann, T. J. Mulder, and J. E. Kallan, "Methodology and assumptions for the population projections of the United States: 1999 to 2100", Popul. Div. U.S. Census Bur., N. 38 , 2000.

[9] R. D. Lee and T. Miller, "Evaluating the performance of Lee-Carter approach to modeling and forecasting mortality," Demography, vol. 38, N. 4 , pp. 537-549, 2001.

[10] K. M. White, "Longevity advances in high-income countries 1955-96”, Popul. Dev. Rev., vol. 28, N. ${ }^{\circ}$, pp. 59-76, 2002.

[11] F. Girosi and G. King, "Understanding the Lee-Carter mortality forecasting method", Gking. Harvard.Edu, 2007.

[12] A. Sklar, "Fonctions de repartition à n dimensions et leurs marges", Publ. l'Institut Stat. l'Université Paris, vol. VIII, pp. 229-231, 1959.

[13] K. Aas, C. Czado, A. Frigessi, and H. Bakken, "Pair-copula constructions of multiple dependence", Insur. Math. Econ., vol. 44, N. 2, pp. 182-198, Apr. 2009.

[14] T. Bedford and R. M. Cooke, "Vines - A new graphical model for dependent random variables", Ann. Stat., vol. 30, N. ${ }^{\circ}$, pp. 1031-1068, 2002.

[15] D. Kurowicka and R. M. Cooke, "Distribution - free continuous Bayesian belief nets", in Modern Statistical and Mathematical Methods in Reliability, vol. 10, World Scientific Publishing Company, 2005, pp. 309-323.

[16] K. Dowd, Measuring Market Risk. Chichester: John Wiley \& Sons, 2005. 